\title{
Nondestructive indication of fatigue damage and residual lifetime in ferromagnetic construction materials
}

\author{
by \\ Ivan TOMÁS̆ ${ }^{\mathrm{a}}$, Ondřej KOVÁŘÍK ${ }^{\mathrm{b}}$, Gábor VÉRTESY ${ }^{\mathrm{c}}$, and Jana KADLECOVÁa \\ ${ }^{a}$ Institute of Physics ASCR, Na Slovance 2, Prague 18221, Czech Republic \\ ${ }^{b}$ Department of Materials, Faculty of Nuclear Sciences and Physical Engineering, Czech \\ Technical University in Prague, Trojanova 13, Prague 120 00, Czech Republic; \\ ${ }^{c}$ Research Centre for Natural Sciences, Institute of Technical Physics and Materials Science, \\ H-1121 Budapest, Konkoly Thege Miklós út 29-33, Hungary
}

Key words: fatigue, residual lifetime, magnetic NDE, ferromagnetic construction materials, Magnetic adaptive testing

\begin{abstract}
A new revolutionary attitude towards investigation of fatigue damage in cyclically loaded steel samples is reported. The measurement is based on the method of Magnetic adaptive testing, which - in contrast to traditional magnetic hysteresis investigations - picks up the relevant information from systematic measurement and evaluation of whole minor magnetic hysteresis loops and their derivatives. Satisfactory correlations between nondestructively measured magnetic descriptors and actual lifetime of the fatigued material were found. The presented method is able to serve as a powerful tool for indication of changes, which occur in structure of the inspected objects during their industrial service lifetime, as long as they are manufactured from ferromagnetic materials.
\end{abstract}

\section{Introduction}

Owing to their beneficial mechanical properties, ferromagnetic materials are widely used in fields such as railways, pipelines, power stations, pressure vessels, petrochemical engineering, and even aerospace. Many ferromagnetic components are subjected to alternating load in service, which often causes their structural failure as a result of fatigue [1 4]. Fatigue evaluation and residual lifetime assessment are challenging issues with a high profile in industry. Nearly $90 \%$ of industrial component failures take place due to fatigue that occurs without warning. In order to check up health of ferromagnetic construction materials, it is important to timely monitor their fatigue damage, i.e. to detect initiation and propagation of fatigue cracks, and to predict residual lifetime of the constructions.

Fatigue process of a mechanically loaded solid metal object can be commonly characterized by its fatigue lifetime. Generally the fatigue lifetime consist of pre-initiation phase, nucleation phase and three phases of the fatigue crack growth continuously merging into each other. The pre-initiation is characterized by variation of macroscopic mechanic properties of the material, caused by re-arrangements and changes of density of dislocations. The material can soften or harden, the variations can be even non-monotonous. Nucleation or initiation of the fatigue micro-cracks takes place during the nucleation phase. The rearrangement of dislocations can lead to local stress concentrations that give rise to crack nuclei. Localized stress enables the crack nuclei to grow and fatigue micro-cracks are formed 
during stage I of the fatigue crack growth. In stage II, one or few of the micro-cracks develop into macro cracks and start a stable growth, i.e. the crack growth rate is proportional to the stress intensity factor. In stage III, the crack growth becomes unstable, and the crack growth rate is increased until a certain critical crack length is attained. Then the stress concentration factor at the crack tip exceeds the fracture toughness of the material and final breakage of the loaded object takes place.

If any industrial object should be safely protected against its complete breakage, an attitude referred to as a "damage-tolerance philosophy" is frequently applied. This requires application of some indicative techniques, which are able to detect danger of approaching mechanical problems in time, during the starting phase of the fatigue cracks nucleation and spreading already, namely soon enough before the main crack achieves its critical length.

Over the past century, many non-destructive methods have been studied in research of the fatigue damage evaluation. Some of the methods that showed promise for metals and alloys included X-ray diffraction, see e.g. [5], laser diffraction [6], infrared thermography [7], and positron annihilation [8]. At present, non-destructive test methods for detecting fatigue cracks, such as ultrasonic test, eddy current inspection and penetrant inspection, etc., can only find macro-defects of certain sizes. These methods are unsuitable for early warning of the approaching fatigue damage $[9,10]$. As a dynamic detection method, acoustic emission can diagnose early failure, but has some limitations in wide applications. For example, measurements by using this method must be performed under the loading conditions, and measured signals are difficult to be analyzed due to interference by noise [11, 12].

Fatigue damage detecting sensors are also frequently used for early detection of fatigue. For a review of them, see [13]. The primary advantages of these sensors are generally summarized as being small in size, with simple design, ease of preparation, low cost, good metrological characteristics, and a lack of necessity for a permanent connection of complex measuring and recording instruments. On the other hand, the common drawbacks for the application of these fatigue damage sensors in actual products are high environmental sensitivity, low stability, low reliability, as well as low repeatability. Owing to the stringent requirements of industrial products, such as repeatability, reliability, and stability, the fatigue fuse with a small slit and the electrical resistance fatigue gauge are the two most promising and commercially available fatigue sensors for industrial applications. However, until now, only a few of these two types of fatigue damage sensors have been developed commercially and applied in selected industrial areas (e.g. steel bridges).

If the investigated object is ferromagnetic, e.g. steel or cast iron, then certainly one of the promising domains of parameters, which can be used for monitoring the fatigue degradation process, is examination of magnetic state of critical locations of the object. Magnetic measurements are usable NDT techniques for evaluating fatigue damage of ferromagnetic materials, because modifications of density and patterns of dislocations, and formation of persistent slip bands result in magnetic property changes. The main potential magnetic techniques for detecting fatigue damage include measurements of Barkhausen effect (BE) $[14,15]$, magneto-acoustic emission (MAE) [16], methods based on magnetic flux leakage (MFL) and magnetic hysteresis. BE and MAE signals originate from discontinuous magnetization processes occurring during the hysteresis cycle, and they are both strongly influenced by stress and deformations. The disadvantage of these techniques for NDT applications is that BE and MAE signals are generally weak and, therefore, a very sensitive measurement equipment is required and the danger of interfering noise is high.

The metal memory method (MMM) is a relatively new, passive non-destructive testing method based on the magneto-mechanical effect. The fundamentals of the method are elaborated in references $[17,18]$. It is an extended magnetic flux leakage method, similar to the normal MFL but without artificial excitation. Due to the effects of applied load and the 
Earth's magnetic field, spontaneous stray field signals can occur on the surfaces of ferromagnetic materials during their manufacturing process or operation. Its normal component, $H_{\mathrm{p}}(y)$, likewise known as metal magnetic memory signal, can be utilized to evaluate the ferromagnetic materials' degree of damage. Compared with other magnetic methods, MMM is simple and easy to master. Previous studies have shown that MMM can be used for evaluation of changes in microstructure and local stresses, which are introduced to and accumulated in the materials during fatigue [19, 20]. However, until now, the signal quantification is rather difficult owing to complicated factors influencing the MMM signals [21 - 24], and more systematic tests and deeper theoretical support is required. It is still a question whether and how this method can be used to predict the fatigue residual lifetime. In addition, many factors can affect the very weak $H_{\mathrm{p}}(y)$ signals, such as chemical composition of the ferromagnetic steel, shape and size of the ferromagnetic components, and heat treatment conditions, among others. This results in complex distributions of $H_{\mathrm{p}}(y)$ signals in different ferromagnetic structures. In order to facilitate study of the $H_{\mathrm{p}}(y)$ signals on laboratory specimens, a small transverse notch [25] or a small concave depression [26] must be sometimes fabricated on the specimens, in order to create artificial stress concentrators and to localize positions where the fatigue cracks appear and where the signal should be measured.

A typical magnetic method is measurement of magnetic hysteresis loops [27], from which structure-sensitive parameters can be extracted. The traditional magnetic parameters of major hysteresis loop, like coercivity, remanence or maximum permeability, were shown, see e.g. $[28,29]$, to be sensitive with respect to properties variation of ferromagnetic materials during fatigue processes. Describe the fatigue lifetime with a variable $t=<0, T>$, where $t$ can represent actual service lifetime of the object, i.e. number of cycles at a harmonic loading by a constant load, number of start-stop cycles, or similar. Let $T$ be the total lifetime, i.e. that value of $t$, when the applied fatigue destroys the object completely. The traditional magnetic parameters are most sensitive mainly at the beginning of the first (the starting 5\% of the total lifetime, i.e. approximately until $0.05 T$ ) and at the end of the third (the last 10 to $15 \%$ of the total lifetime, i.e. approximately after $0.85 T$ ) phase of the fatigue process [30, 31]. This means the parameters are sensitive during the starting changes of the mechanical properties and then during the main crack spreading. Throughout the major part of the lifetime, i.e. during majority of the middle lifetime of the object, values of the traditional hysteresis parameters are almost constant, which does not offer much opportunity for current monitoring of the advancement of the actual material fatigue damage. Another weakness of the traditional hysteresis parameters as indicators of ferromagnetic construction materials fatigue is the fact, that their starting and final sensitivities are rather pronounced at laboratory ferromagnetic materials (e.g. $\mathrm{Fe}+0.1 \% \mathrm{Cu},[28])$ but they are hardly achieved at common construction steels. Last, but not least, the measurement of the full, major hysteresis curve, from which traditional parameters are determined, requires magnetic saturation of the measured object, which is often anything but straightforward.

Similar behavior [32] of fatigued commercial steel CSN 12021 was also observed with the use of a recently developed hysteresis method, referred to as Magnetic adaptive testing (MAT). MAT is based on systematic measurement and evaluation of minor magnetic hysteresis loops, it was described in details in [33], and potentiality of its application for testing fatigue of ferromagnetic construction materials is shown in the present paper. Actually, the main purpose of the present paper is to illustrate on an experimental example, that by using the multi-parametric character of MAT, and a special proper experimental and evaluation technique, we can provide very adequate current monitoring of advancing fatigue damage in ferromagnetic construction objects. The MAT parameters are easily measured, 
their slight variation during the whole fatigue process is able to yield at least a rough estimate of the exploitable residual lifetime and certainly they can serve as timely indicators of the approaching danger of the lifetime final end.

The applied method and the performed experiments are described in Section 2, their evaluation and results are presented in Section 3 and the so-far evident advantages and disadvantages of the MAT approach will be discussed in Section 4.

\section{Method and Experiments}

Description of the applied method and of the performed experiments is presented in the three following paragraphs. The samples and the way of their loading are illustrated in the first paragraph. The second paragraph is dedicated to the electro-mechanical set-up for the SF-Test, which was adapted and used for this series of experiments. The last paragraph of this Section provides basic information about the employed electro-magnetic system and the method of Magnetic adaptive testing, as it was modified for the fatigue damage application.

\subsection{Samples}

The samples are made of low-carbon steel (S235JR) with two types of heat treatment. The "cold rolled" samples are made of steel sheets recrystallization-annealed $\left(550-700^{\circ} \mathrm{C}\right)$ and slightly cold rolled. The "hot rolled" samples are made of $4 \times 40 \mathrm{~mm}^{2}$ bars, hot rolled (850$1100^{\circ} \mathrm{C}$ ) without annealing. The shape of the specimens is shown in Fig.1, with the crosssection of the middle narrow part $4 \times 20 \mathrm{~mm}^{2}$. The bottom head of the sample is fixed in a solid vice with non-magnetic jaws. A small ferromagnetic armature, which is mounted at the top head of the sample, helps to the sample to be periodically bent back and forth in a controlled way by a couple of deflection electromagnets. (See also Fig.2.)

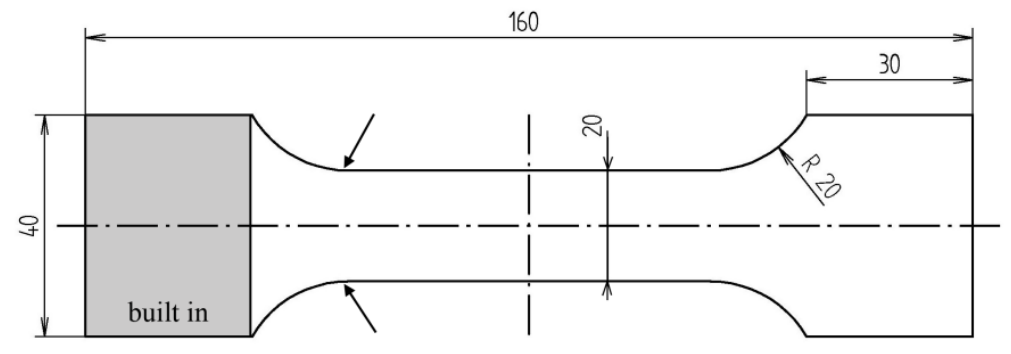

Fig.1 The standard shape sample made of low-carbon steel. The arrows mark the crack initiation area. The dimensions are in millimeters. The sample thickness is $4 \mathrm{~mm}$.

The specimen is ferromagnetic, it is flat, and it can be magnetized for the MATmeasurement by a short solenoid placed over the critical volume, where the fatigue cracks are initiated in the sample. This critical area is the neck, close to the fixed bottom head of the sample (See also Figs.1 and 2). An easy magnetization of the sample during the MAT measurement is helped by a couple of passive yokes (two transformer C-yokes), which are pressed by a special plastic holder to the flat surfaces of the sample during the magnetic testing.

\subsection{Mechanical loading of the samples: SF-Test}

Cyclic loading of the samples takes place at an adapted SF-Test facility, see e.g. [34, 35], shown in Fig.2. The bottom-fixed sample is loaded by swinging back and forth its top part (which is equipped with a small armature to increase the deflection forces and to decrease 
the resonance frequency of the system) by an ac electromagnetic field induced by two electromagnets. Frequency of the electromagnetic field, i.e. the excitation frequency of the specimen, is kept equal to the resonance frequency of the sample-armature system. The excitation frequency (in our case around $80-90 \mathrm{~Hz}$ ) is controlled by maintaining a constant phase shift between the excitation force and the specimen deflection. The deflection amplitude of the free top end is measured by a laser distance sensor. A PID regulator is used to control the excitation force amplitude in order to keep the free end deflection constant. For the performed experiments, the free end amplitude of $4 \mathrm{~mm}$ was chosen, which resulted in fatigue lifetimes of the tested samples in the order of several hundred kilocycles.

The gradually developing fatigue damage is reflected by changes of the resonance frequency. During the pre-initiation phase, the cyclic hardening or softening of the material influences the specimen stiffness. During the crack initiation and propagation phases, the fatigue cracks effectively reduce cross-section of the specimen, which results in an accelerated decrease of the resonance frequency. Level of the fatigue damage of the samples can thus be estimated from the resonance frequency variation.
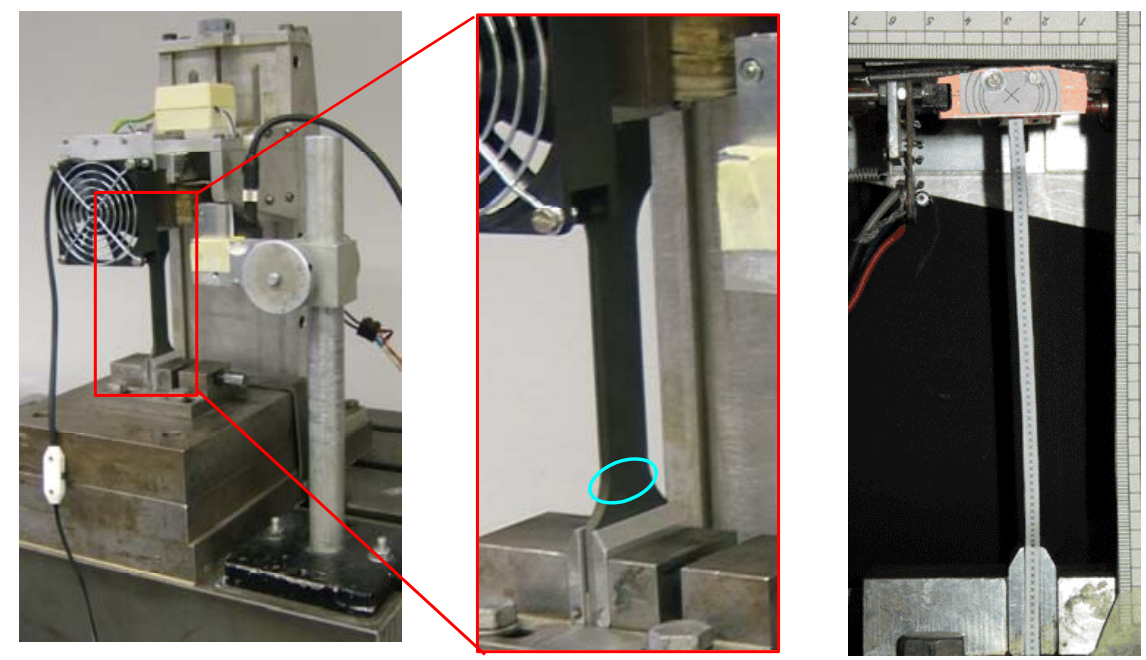

Fig.2 The electro-mechanical part of the SF-Test system for cyclic loading of standard samples by mechanical bending. The fatigue cracks are initiated in the area of maximum stress marked by the colored ellipse in the middle photo.

\subsection{Magnetic measurement of the samples: MAT}

Physical principles, experimental application and ways of evaluation of results of MAT are described in most details in the first two paragraphs of [33]. For the purpose of this paper it is probably enough to point out that Magnetic adaptive testing proceeds by magnetization of the tested ferromagnetic object by slowly oscillating magnetic field with constant absolute value of its rate of change and with amplitude, which starts at a chosen minimum and is increased by a constant step after each period, until magnetic saturation of the sample is achieved or at least approached. A voltage signal is induced in a pick-up coil wound around the magnetized circuit. The recorded data constitute a large family of minor loops of differential permeability, $\mu$, of the magnetized circuit, a part of which is formed by the investigated object. The data are processed and interpolated into a large set of descriptors, 
$\mu(I, A, t)$, of the magnetic state of the magnetized circuit, each of them indexed by a triplet of parameters. They are the actual magnetizing current, $I$, the actual current amplitude, $A$, of the minor loop and the actual fatigue degradation lifetime, $t$, of the sample. Each sequence of the descriptors, which have their parameters $I=I_{i}$ and $A=A_{j}$ constant and only $t$ is varied from $t=0$ up to $t=T$, and which is normalized by value of the corresponding descriptor at $t=0$ (i.e. of the "virgin" or reference sample), is called a normalized degradation function $\mu\left(I_{i}, A_{j}, t\right)$ of the fatigue degradation lifetime, $t$, of the sample. The produced degradation functions are compared with each other and the most sensitive and/or the most indicative of them are picked up for the best indication of the fatigue degradation process. The degradation functions, which were created straight from the induced signal, are usually labeled as the $\mu$ degradation functions. However, for the purpose of indication of the fatigue, degradation functions based on the derivative $\mathrm{d} \mu / \mathrm{d} I=\mu$ ' proved to be substantially more indicative. They are used in the next figures and they are referred to as the $\mu$ '-degradation functions, $\mu^{\prime}\left(I_{i}, A_{j}, t\right)$.

The MAT-measurement of our samples proceeded in such a way, that always after a chosen number of loading cycles a short solenoid containing both the magnetizing and the pick-up coils was placed over the critical part of the sample, a couple of passive soft magnetic yokes was pressed to the sample so, that they and the sample created a closed magnetic circuit, and one family of the minor permeability loops was recorded. Then the yokes were taken away and a next number of loading cycles were applied. Fig. 3 shows the sample with the yokes and with the yoke-holder during the magnetic measurement.

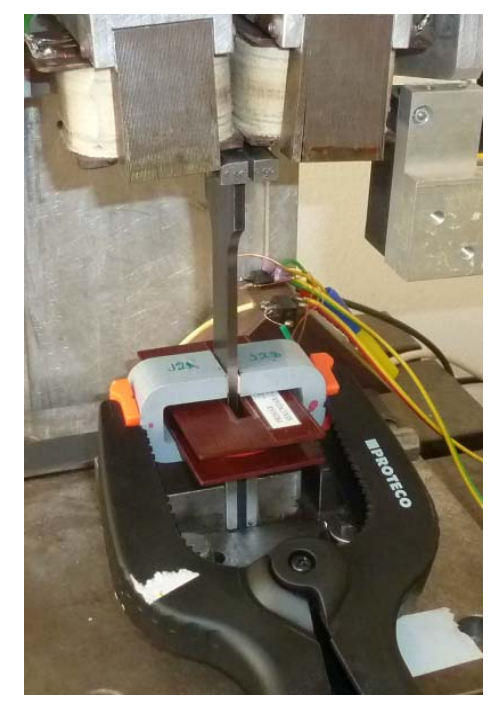

Fig.3. The critical part of the sample is covered by the magnetizing/sensing solenoid and by a couple of passive soft yokes pressed to the surface of the sample with a plastic spring holder. All is fixed at the bottom in a vice with non-magnetic jaws. The couple of deflection electromagnets can be seen at top of the figure.

\section{Results}

For purposes of illustration in this paper, a single sample made of cold rolled S235JR steel was chosen for presentation of a typical MAT-measurement of the fatigue damage process. The selected sample was fatigued and measured as described in Section 2. The continuous recording of the resonance frequency supplied an immediate controlling information about approximate mechanical state of the sample, and allowed to focus in more details on those parts of the sample lifetime, when its mechanical state varied faster than at the 
middle, slower-change, lifetime part. The magnetic measurement was carried out first on the virgin (not fatigued) sample, and then approximately after each 10 kilocycles $(\mathrm{kc})$ at the starting and at the ending parts of the fatigue lifetime, and after each $20 \mathrm{kc}$ in the middle part.

The sample was magnetized by a short solenoid with 200 turns of wire $\varnothing 0.5 \mathrm{~mm}$, and the pick-up coil (wound on the same support) had 300 turns of $\varnothing 0.15 \mathrm{~mm}$ wire. Each of the two passive yokes had cross-section $10 \times 25 \mathrm{~mm}^{2}$, inner distance between the legs $15 \mathrm{~mm}$, and $36 \mathrm{~mm}$ outside height in the bow. Nonmagnetic spacers with thickness $0.095 \mathrm{~mm}$ were placed between the faces of each yoke and the sample surface. (Role of these spacers is explained and discussed in Section 4.) The magnetizing current amplitude started at $A=0.02$ Amp and continued by steps of 0.02 Amp until the maximum amplitude 0.8 Amp was attained. The magnetizing current was varied in a triangular way, with the rate of change kept always $\pm 0.4 \mathrm{Amp} / \mathrm{s}$.

Careful evaluation of shapes and sensitivities of the complete set of the $\mu^{\prime}\left(I_{i}, A_{j}, t\right)$ degradation functions, created as described in Section 2 (or in more details in [33]), revealed that starting from the minor loops amplitude $A_{j} \geq 0.4 \mathrm{Amp}$ the $\mu$ '-degradation functions offered results, which were very well applicable for the purpose, and practically identical with one another. In Fig. 4 some typical $\mu^{\prime}\left(I_{i}, A_{j}=0.5 \mathrm{Amp}, t\right)$-degradation functions computed from derivative of the induced signal at the minor loop with amplitude $A_{j}=0.5 \mathrm{Amp}$, for a number of immediate values, $I_{i}$, of the sweeping magnetizing current, are plotted. They will be referred to as $\mu_{I i}$ ' $(t)$ in the following text.

A number of $I_{i}$ gives many applicable $\mu_{I i}{ }^{\prime}(t)$-degradation functions, which can be successfully applied for indication of the fatigue-induced changes in the measured sample. They are either non-monotonous, as those with $I_{i}=-0.11 \mathrm{Amp}$ and those with $I_{i}=0 \mathrm{Amp}$ or they can be monotonous as those with $I_{i}=-0.21 \mathrm{Amp}$ and those with $I_{i}=+0.15 \mathrm{Amp}$. The normalized curve of the resonance frequency is also plotted. Evidently the start of the accelerating curvature of the $\mu_{I i}$ ' $(t)$-degradation functions is situated at about very similar actual lifetime, $t$, or even sooner, then that of the resonance frequency.

Fig. 5 was added for a better understanding of the construction of the $\mu_{I i}{ }^{\prime}(t)$ degradation functions. Shapes of the $\mu_{I i}{ }^{\prime}(t)$-degradation functions, for individual values of $I_{i}$, can be constructed from the varied profiles of the $\mu^{\prime}\left(I, A_{j}=0.5 \mathrm{Amp}, t_{k}\right)$ curves for the explicitly shown $t_{k}$ values corresponding to the magnetic measurements at each stop of the fatigue process. 


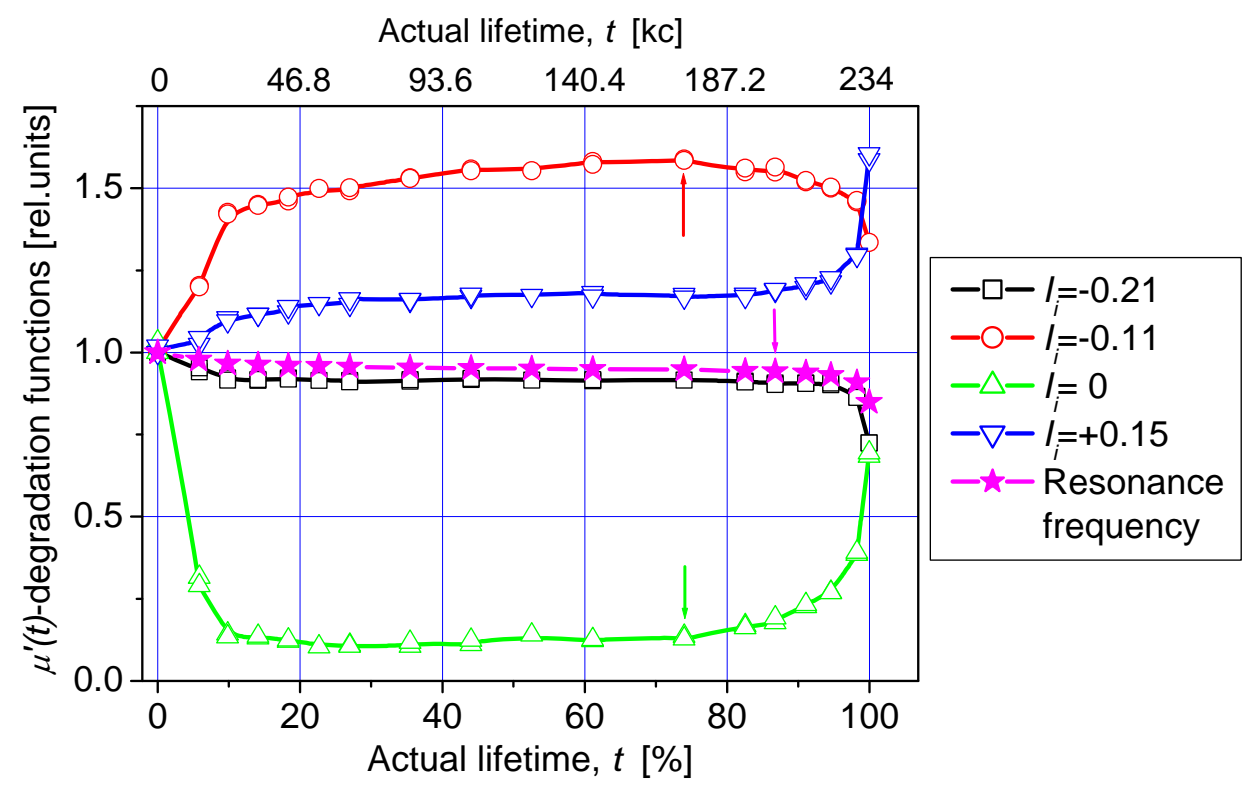

Fig.4 Examples of $\mu_{I i}{ }^{\prime}(t)$-degradation functions for several values of $I_{i}$ obtained from the minor loop with amplitude $A_{j}=0.5 \mathrm{Amp}$. The curves for $I_{i}=-0.11 \mathrm{Amp}$ and $I_{i}=0 \mathrm{Amp}$ are non-monotonous, the curves for $I_{i}=-0.21 \mathrm{Amp}$ and $I_{i}=+0.15 \mathrm{Amp}$ are monotonous. The curve of stars presents behavior of the normalized resonance frequency of the sample, which was continuously measured by the SF-Test equipment during the whole fatigue process. Its shape is very close to the $\mu_{I i}$ ' $(t)$-degradation functions with $I_{i}=-0.21 \mathrm{Amp}$ (and to several others - not shown here - close to $\left.I_{i}=-0.21 \mathrm{Amp}\right)$. Generally, all the $\mu_{I i}{ }^{\prime}(t)$-degradation functions presented in the figure achieve their most notable feature (the fast upward or downward curvature, see the arrows for the $\mu_{-0.11}{ }^{\prime}(t)$ - and the $\mu_{0}{ }^{\prime}(t)$-curves) at or even sooner than the start of the curvature acceleration of the resonance frequency (see the relevant arrow), which in its turn indicates start of the critical crack spreading through the sample.

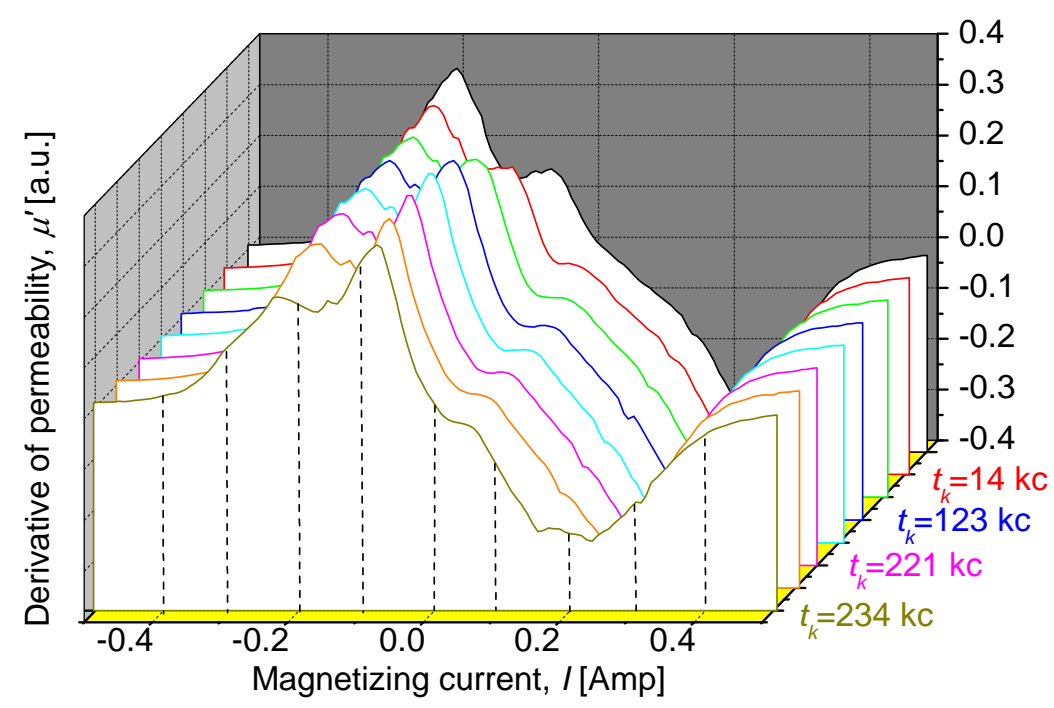


Fig.5 Half-loops of the first derivative of differential permeability $\mu$ ' $\left(I, A_{j}=0.5 \mathrm{Amp}, t_{k}\right)$ for $0 \leq t_{k} \leq 234 \mathrm{kc}$ (only the half-loops corresponding to the increase of the magnetizing current from the negative values towards the positive ones) plotted in a "waterfall" type 3D-graph. The graph allows to see changes of $\mu^{\prime}(I)$ during the whole fatigue process of the sample. Horizontal scale at the right-hand side of the figure represents the actual lifetimes of every second of the plotted curves. (These half-loops are identical with the relevant halves of the loops plotted in Fig.8 in Section 4.)

\section{Discussion}

It was stated in the Introduction already, that microstructure of the cyclically loaded solid material undergoes modifications, which re-shape general pattern of dislocations and substantially change the dislocation density. These changes cause redistribution of inhomogeneous local stresses and micro-strains, which eventually may lead to occurrence of micro-cracks and even to the final main crack. Magnetic properties of ferromagnetic materials are very sensitive to distribution of internal stresses. This effect was investigated by many researchers, starting from 1930 and continuing with increasing understanding and with a gradually improving theoretical description until now, see e.g. [19, 20, 31, 36 - 38]. Process of magnetization of each ferromagnetic material proceeds mainly via irreversible motion of magnetic domain walls and the dislocations pin domain walls through the magnetoelastic coupling. Therefore it is not surprising that even the macroscopic behavior of any ferromagnetic cyclically loaded object responses to modifications of the dislocations pattern/density and eventually even to formation of micro-cracks, which - in their turn - are accompanied by appearance of an extra internal demagnetization.

However, as mentioned in Section 1 and in Paragraph 2.3, the traditional magnetic hysteresis parameters vary with fatigue lifetime of construction steel samples only very slightly. Nevertheless, at some ranges of the magnetization process, first derivative of the differential permeability can show very well applicable changes. Figs.6, 7 and 8 illustrate this situation for the used sample. They present very small differences of the traditional magnetic hysteresis parameters $H_{C}, B_{R E M}$, and $\mu_{M A X}$ in Figs.6 and 7. Actually, the hysteresis loops in Fig. 6 look almost identical with each other for lifetimes of the sample from $t_{k}=0$ up to $t_{k}=T$. The only differences - if any - can be observed in the ranges of the loops "knees". Fig.7 suggests, that it is really so, but the differences of the measured signal values (i.e. of values of $\mu(I))$ are still minute. However, the slopes of $\mu(I)$ are certainly different from one another and this is confirmed by plots of $\mu^{\prime}(I)$ in Fig. 8 . 


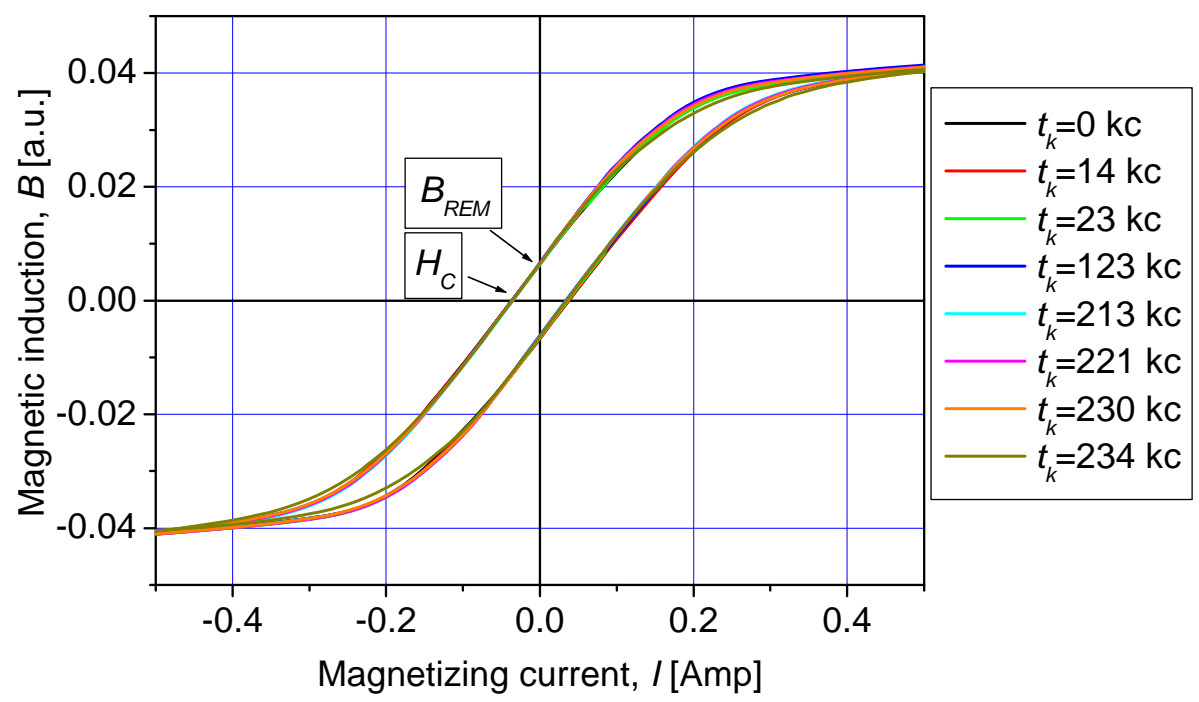

Fig.6 Hysteresis loops of magnetic induction, $B(I)$, for 8 values of the actual lifetime, $t_{k}$, of the sample. These loops were computed by integration of the measured induced signals, halves of which are plotted in Fig.7. It is evident that both coercivity, $H_{C}$, and remanent magnetic induction, $B_{R E M}$, varied during the whole lifetime of the sample only very little.

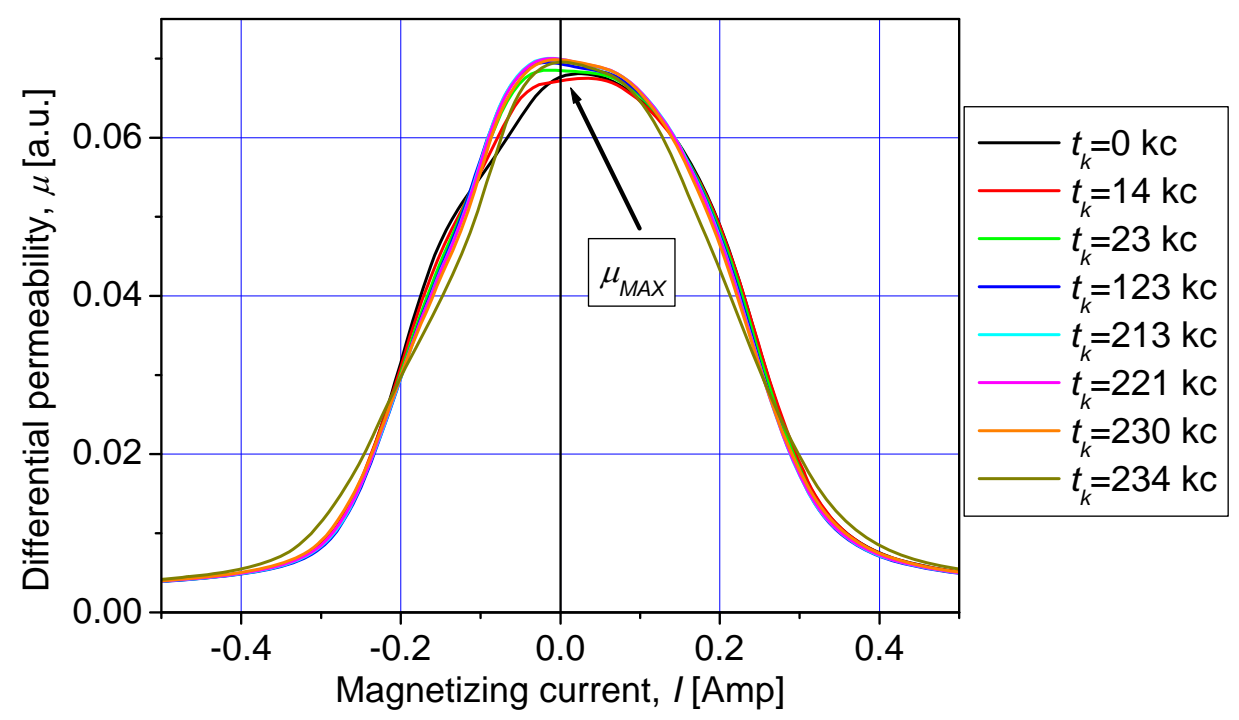

Fig.7 Directly measured half-loops of the induced signal, proportional to the differential permeability, $\mu(I)$, of the tested magnetic circuit, plotted for the same 8 values of the sample lifetime as in Fig.6. In order to make the figure more lucid, only halves of the signal loops are plotted, namely those which correspond to the increase of $B$ with the increase of $I$ (i.e. corresponding to the bottom branches of the hysteresis loops in Fig.6.) Here it is evident that the maximum permeability, $\mu_{M A X}$, was slightly varied during the sample lifetime. However, the variations can be much better observed in slope of the curves at certain ranges of the magnetizing current. i.e. at certain ranges of the first derivative of the measured signal. This is presented in Fig.8. 


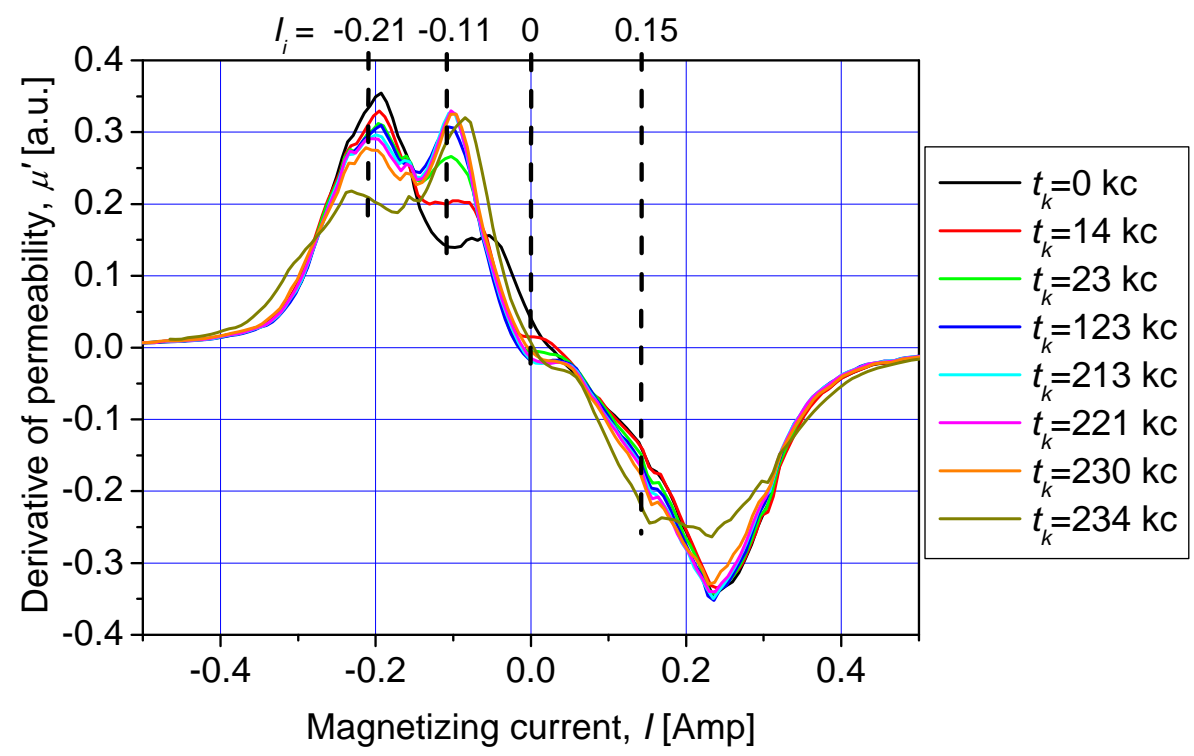

Fig.8 Half-loops of the first derivative of the induced signal, $\mu^{\prime}(I)$, of the tested magnetic circuit, corresponding to the half-loops of the signal plotted in Fig.7. The shapes and values of $\mu^{\prime}(I)$ are very distinctly varied with the advancing sample lifetime at some ranges of the magnetizing current. The well indicative $\mu$ '-degradation functions shown in Fig. 4 of Section 3 were taken just for the $I_{i}$ values shown in this figure by dashed vertical lines. Also compare the curves plotted here with those plotted in Fig.5.

As mentioned above, the traditional magnetic parameters are sensitive mainly during the starting changes of the mechanical properties and then during the main crack spreading, when the investigated object approaches the end of its total lifetime. This was observed before, e.g. in [28] and others. The $\mu$ '-degradation function for $I_{i}=-0.11 \mathrm{Amp}$ in Fig.4 closely corresponds to the results of the traditional measurements based on major hysteresis loops. Evidently, the MAT data are not in contradiction with those results but yield more parameters by means of a whole set of the $\mu$ '-degradation functions. Some of them are monotonous as functions of the actual lifetime, $t$, some are not, and they together open a way to a more complex and substantially more reliable characterization of the material degradation, than the few classical parameters only. It is an important goal of MAT to look for the most proper choice of a number of applicable parameters, mutually supporting and completing one another, from the large available measured data pool.

The curves in Fig. 4 represent the four main types of the $\mu$ '-degradation functions, which are available for indication of the approaching end of lifetime of the fatigued objects. Evidently, they are not singular and degradation functions with $I_{i}$-values close to those explicitly introduced in Fig.4 differ from the shown curves only a little. The explicitly introduced $I_{i}$-values generally indicate the middle of limited ranges of mutually similar functions.

Each point of each degradation function in Fig.4 was measured several times. The observed scatter of experimental values was very small (even though the sample surface was not polished and it corresponded to the ordinary industrial rolling) and the corresponding error bars do not exceed size of the plotted point symbols. This observed minute scatter speaks about a high reliability of the measurement and about a trustworthy indication of the tested object's fatigue degradation. It is especially important in the region of the fast upwards or 
downwards curvature of the $\mu$ '-degradation functions, when the object approaches end of its lifetime.

It was a great advantage for the whole experiment that the well-understood and many times checked-up SF-Test could be used throughout the measurement as an immediate indicator of the advanced fatigue damage lifetime and that the magnetic MAT-measurement could be continuously checked and controlled by this knowledge. The SF-Test informs about the advancing fatigue damage of the sample via variation of the sample resonance frequency. Unfortunately, of course, the SF-Test is not easily applicable to industrially interesting construction details, as their resonance frequency can be measured only in very special configurations, if at all.

Testing of the fatigue damage caused by cyclic bending of flat samples is experimentally very friendly. Firstly, the applicable frequencies are around $80 \mathrm{~Hz}$, and application of several hundred thousand of cycles does not last unpleasantly long time (this is in contrast with frequently used tension/compression fatigue experiments in electro-hydraulic loading machines, where the maximum achievable frequency hardly attains $10 \mathrm{~Hz}$ ). And secondly, the flat surface of the samples makes it simple - just with a pair of commercial soft yokes - to create a good magnetic circuit for the necessary strong enough magnetization of the sample (also in contrast with the common tension/compression loading, where the usual shape of the samples is cylindrical and closing of a magnetic circuit is much more difficult).

Actually, even the "simple" magnetic contact of flat yoke faces on a flat surface of the tested object can bring about problems, as quality of the magnetic contact is often difficult to be reproduced without a rather large fluctuation. It was shown in [39], however, that this problem is very well solved with the aid of a thin non-magnetic spacer placed between the yokes faces and the object surface. A non-magnetic spacer $0.095 \mathrm{~mm}$ thick was used in the presented example. The spacer minimizes the fluctuation, decreases the induced signal and also modifies its shape. We plan to look systematically for optimum applicable thickness of the spacers in our next research program. Preliminary results of detailed optimization of the experimental conditions reveal existence of shapes of $\mu$ '-degradation functions with even steeper slope in the middle part of the damaged object's lifetime than those plotted as an example in Fig.4, enabling to follow progression of the object's lifetime almost throughout the complete process of its fatigue damage, see [40].

The yokes used in the presented example were passive: the sample was magnetized and "sensed" by coils wound around its body. It is sure, that this configuration is very special and probably will be applicable only in rare industrial cases, when such a configuration is allowed by the shape and position of the tested construction. In other cases, however, active magnetizing/measuring yokes - inspection heads - can be used. These have the magnetizing and the pick-up coils wound on their bodies, and magnetization and picking up of the induced signal of the magnetic circuit, one part of which is the tested object, is performed by the coils on the yoke. In principle, objects of any size and shape can be inspected with the aid of such an inspection head. We started testing active inspection heads and plan to investigate systematically their advantages and disadvantages.

The measured correlation between MAT parameters and the actual fatigue lifetime does not depend on the applied method of the object magnetization. This important feature, which makes industrial use of MAT actually applicable, was discussed in [41], where influence of the magnetizing and the pick-up techniques on the optimum magnetic descriptors of MAT was studied by measuring a series of gradually degraded individual steel samples. The same series of flat samples were magnetized by an attached active inspection head and by an open solenoid. It was found that qualitatively the same relationship was obtained, regardless of the actual type of the experimental conditions. The sensitivity depends on the technique of magnetization, but there are no functional differences between application of different types 
of magnetizing yokes and on exact positions of the pick-up coils. An open magnetization circuit yields lower sensitivity due to the strong demagnetizing effect of the samples shape, but even in this case a corresponding relationship can be found.

The very first experiments with the fatigue indication by MAT were carried out using the full analysis of the measured data. Those data were obtained during magnetization of the samples in the broadest range of the magnetizing current amplitudes, from an achievable minimum up to maximum amplitudes, which almost saturated the samples. Analysis of the sensitivity maps (see [32] for details on sensitivity maps) revealed, that the very low amplitudes do not give applicable results, and that it is advantageous and sufficient to use larger amplitudes only. As an approximate "rule of thumb" the samples do not need to be magnetized by magnetizing fields as low as those that do not create well-developed "knees" on the hysteresis loops. The sensitivity maps (not shown in this paper) reveal also, however, that neither too large magnetizing amplitudes (close to saturation) are required, as starting from a certain value (e.g. $A_{j} \geq 0.4$ Amp for the used sample, the applied magnetizing coil and the yokes) all the mutually corresponding degradation functions coincide.

Application of large enough "medium" magnetizing amplitudes present an additional small experimental advantage: It is not necessary to demagnetize the samples artificially (e.g. by a decreasing ac field) before each MAT measurement, in order to insure identical starting magnetic conditions of the sample. No differences were observed between data obtained on properly demagnetized and on not properly demagnetized samples. Evidently, shape demagnetization of the samples, caused just by removing the magnetic-circuit-closing yokes from the sample surface, was here enough.

Within the paper, we mostly speak about the magnetizing current during the MAT measurement, even though values of the magnetizing field would be more descriptive and certainly more general. However, to measure the magnetizing current is easy and to measure the magnetizing field inside the sample is difficult. As magnetic adaptive testing offers only relative data anyway, application of the same experimental conditions at one series of measurements (the same coils, yokes, spacers, etc.) ensures, that use of the easily measurable current is quite appropriate for simplification of the measuring set-up and it gives perfectly usable results. As discussed above, application of a non-magnetic spacer between yoke and sample guarantees, that even the quality of magnetic contact of a repetitively artificially closed magnetic circuit can be considered to be a repeatable experimental condition within one series of measurements.

As stated above, samples available for the presented paper were produced from low carbon steel S235JR. Two forms of the steel were investigated, hot rolled flat rod (hot rolled specimens) and cold rolled sheet (cold rolled specimens). The processing of the steel influenced its microstructure, its residual stress field, and consequently its fatigue life. The typical fatigue life, $T$, of the cold rolled samples was about $250 \mathrm{kc}$, whereas the hot rolled samples had the fatigue life about twice as large, at the same deflecting conditions. As the MAT response of the cold rolled samples was more pronounced (at the applied experimental conditions), we choose one cold rolled sample for the presented illustration.

It is recommended in many applications of MAT first to measure completely a reference sample/object. This object should be of the same material, of the same shape and should be degraded by the same processes as the unknown next objects, the state of actual degradation of which is planned to be determined in future. The reference object is measured in details during the whole process of its degradation and its recorded MAT-data form a calibration curve (and/or a set of calibration curves), which are corresponding data of the unknown objects then compared with. Such an approach can be easily applied at the fatigue-testing MAT experiments on specially shaped and plentiful samples, when the first basic information about the MAT applicability on the problem is investigated. Such an approach was, of course, 
also used in the experiments, which is the present paper based on. However, a corresponding reference sample can hardly be prepared and tested up to the end of its fatigue life, if we are supposed to investigate a real industrial object or construction. Then, another approach will be necessary, namely the tested object should be measured at the suspected most fatigue-exposed location(s) - at stress concentrators - repeatedly and regularly, always after a reasonably estimated increment of the load cycles. The measured $\mu$ '-degradation functions should be thus constructed point-by-point from those regular repeated measurements during the whole object's lifetime. It is probably not necessary to emphasize, that all the mutually corresponding measurements must be always done at the same locations of the object and at the same experimental conditions, of course. The start of fast change of the curvature of the $\mu$ '-degradation functions (upwards or downwards, according to their type) draws attention of the human operators to the approaching end of the object's lifetime and to the possible danger. Fig. 4 shows the fast change of the curves curvature appears at about $t_{k} \approx 0.8 T$ or even sooner (see e.g. the $\mu^{\prime}$-curves for $I_{i}=-0.11 \mathrm{Amp}$ and $I_{i}=0 \mathrm{Amp}$ ). This is about, or even sooner than, when nucleation of the main crack is indicated by the fast change of curvature of resonance frequency in the SF-Test. Based on our presently carried out experiments, we rightfully suspect this behavior of the $\mu$ '-degradation functions to be typical for all the fatigued ferromagnetic objects and expect the fast increase of their curvature to be applicable as a reliable indication of the approaching end of lifetime of many industrial objects. However, similar and other experiments are presently running and information about them will be published as soon as possible.

It is worth of mentioning, that close to the end of lifetimes of the investigated objects, the qualitative types of correlation between magnetic parameters and $t$, (no matter if obtained from classical coercivity measurement, from MAT or from MMM) are very similar to each other. In classical fatigue magnetic measurements on $\mathrm{Fe}+0.1 \% \mathrm{Cu}$ (see [28]), in fatigue laboratory tests of train axles (see [26]), or in our results on samples of both cold and hot rolled S235JR, always a sudden change of values of the indicative parameters was observed, when the end of lifetime was approaching. It suggests that magnetic methods properly reflect structural modifications, which happen in the inspected fatigued material. Existence of this common good correlation evidently supports reliability and next applicability of magnetic nondestructive testing methods, regardless of the actual choice of method. The choice of the most suitable way of measurement must be made by taking into account the actual conditions of the task to be solved, the reachable signal-to-noise ratio, and frequently also the mutual multi-parametric support of simultaneously obtained parameters as is the case e.g. at MAT.

The best result of the magnetic measurement can be evidently expected if the magnetic inspection is performed at such a locality of the fatigued object, where the first appearance of the main crack is expected, namely at a stress concentrator position. Preliminary experiments showed, that modifications of the material microstructure can be magnetically observed even at positions at some distance from the concentrator. However, no systematic research of such a distance-dependence was realized yet, and at the moment we are not able to give any quantitative description of such situations. More detailed investigation is necessary and we plan to look into the problem in our next experiments. At this moment, however, the primary recommendation is either to locate the stress concentrator position, or even - perhaps - to create an artificial stress concentrator on the investigated object and to perform the measurement just there.

\section{Conclusions}

Damage of flat low-carbon steel samples subjected to resonance bending fatigue test was investigated. A recently introduced nondestructive method of magnetic adaptive testing 
(MAT), which is based on systematic measurement and evaluation of minor magnetic hysteresis loops and their derivatives, was applied. We showed by an experimental example, that multi-parametric data acquired by MAT can provide a way to complex current monitoring of advancing fatigue damage in ferromagnetic construction objects. The presented illustrative example explained main features of the measuring and evaluation procedures and demonstrated, that by combination of several types of the monitoring experimental curves referred to as $\mu$-degradation functions - a reliable prediction of the approaching end of lifetime of the tested object can be obtained at the process time when more than about $20 \%$ of the undisturbed exploitable lifetime is still available.

More experiments, aiming at verification and optimization of the MAT indication of advancing fatigue damage in ferromagnetic construction materials and objects are under work and will be currently published.

\section{Acknowledgement}

The authors are indebted to the financial support by the project No.TA02011179 of the Technology Agency of the Czech Republic, and modification of the fatigue test device and methodology was supported by project No.P108/12/1872 of the Grant Agency of the Czech Republic.

\section{References:}

[1] J. Pesicka, R. Kuzel, A. Dronhofer, G. Eggeler, The evolution of dislocation density during heat treatment and creep of tempered martensite ferrite steels, Acta Mater. 51 (2003) 4847-4862.

[2] Li Nian, Du Baiping, The effect of low-stress high-cycle fatigue on the microstructure and fatigue threshold of a 40Cr steel, Int. J. Fatigue 17 (1995) 43-48.

[3] A. Gilanyi, K. Morishita, T. Sukegawa, M. Vesaka, K. Miya, Magnetic nondestructive evaluation of fatigue damage of ferromagnetic steels for nuclear fusion energy systems, Fusion Eng. Des. 42 (1998) 485-491.

[4] Zheng Xiulin, On some basic problems of fatigue research in engineering, Int. J. Fatigue 23 (2001) 751-766.

[5] M.A.S. Torres, H.J.C. Voorwald, An evaluation of shot peening, residual stress and stress relaxation on the fatigue life of AISI 4340 steel, Int. J. Fatigue, 24 (2002) 877.

[6] A. Kato, M. Hayashi, Fatigue life estimation of steel using laser speckle sensor, NDT \& E International, 32 (1999) 139-145.

[7] M.P. Luong, In: Fatigue evaluation of metals using infrared thermography, Published by Society of Photo-Optical Instrumentation Engineers: Singapore, 2001, p.297.

[8] Y. Kawaguchi, N. Nakamura et al., Non-destructive evaluation of fatigue damage in type 316 stainless steel using positron annihilation lineshape analysis, Materials Transactions 43 (2002) 727.

[9] J. Spanner, G. Selby, Sizing stress corrosion cracking in natural gas pipelines using phased array ultrasound, NDE Eng. 22 (2002) 68-71.

[10] G. Dobmann, L. Debarberis, J.-F. Coste, Aging material evaluation and studies by nondestructive techniques (AMESNDT) - a European network project. Nucl. Eng. Des. 206 (2001) 363-374.

[11] J. Holt, D.J. Goddard, I.G. Palmer, Methods of measurement and assessment of the acoustic emission activity from the deformation of low alloy steels, NDT Int. 14 (1981) 49-58. 
[12] M.A. Abdullah, M. David, A comparative experimental study on the use of acoustic emission and vibration analysis for bearing defect identification and estimation of defect size, Mech. Syst. Signal Process. 20 (2006) 1537-1571.

[13] P. Wang, T. Takagi, T. Takeno, H. Miki, Early fatigue damage detecting sensors-A review and prospects, Sensors and Actuators A, 198 (2013) 46-60.

[14] S.P. Sagar, N. Parida et al., Magnetic Barkhausen emission to evaluate fatigue damage in a low carbon structural steel', Int. J. Fatigue 27 (2005) 317.

[15] C.C.H. Lo, F. Tang et al., Monitoring fatigue damage in materials using magnetic measurement techniques, J. Appl. Phys. 85 (1999) 4595-4597.

[16] D.C. Jiles, Review of magnetic methods for nondestructive evaluation, NDT Int. 21 (1988) 311-319.

[17] A.A. Doubov, A study of metal properties using the method of magnetic memory. Metal Sci. Heat Treat. 39 (1997) 401-402.

[18] A.A. Doubov, A technique for monitoring the bends of boiler and steam-line tubes using the magnetic memory of metal, Therm. Eng. 48 (2001) 289-295.

[19] L. Li, S. Huang et al., Research on magnetic testing method of stress distribution, Transactions of Nonferrous Metals Society of China (English Edition), 12 (2002) 388-391.

[20] L. Li, S. Huang et al., Magnetic field abnormality caused by welding residual stress, J. Magn. Magn. Mater. 261 (2003) 385-391.

[21] Dong Lihong, Xu Binshi, Dong Shiyun, Chen Qunzhi, Metal magnetic memory testing for early damage assessment in ferromagnetic materials. J. Cent. South Univ. Technol. 12 (2005) 102-106.

[22] Dong Lihong, Xu Bin-shi, Dong Shi-yun et al., Study on the magnetic memory signals of medium carbon steel specimens with surface crack precut during loading process, Rare Metals 25 (2006) 431-435.

[23] Dong Lihong, Xu Bin-shi, Dong Shi-yun et al., Investigation of metal magnetic memory signals from the surface of low-carbon steel and low-carbon alloyed steel. J. Cent. South Univ. Technol. 14 (2007) 24-27.

[24] Dong Lihong, Xu Bin-shi, Dong Shi-yun et al., The effect of axial tensile load on magnetic memory signals from the surface of medium carbon steel, Chin. J. Mater. Res. 20 (2006) 440-444.

[25] Dong Lihong, Xu Binshi, Dong Shiyun et al., Monitoring fatigue crack propagation of ferromagnetic materials with spontaneous abnormal magnetic signal, Int. J. Fatigue 30 (2008) 1599-1605

[26] Chen Xing, Li Luming, Hu Bin, Cui Xiaojie, Deng yuanhui, Yang dezhi, Yang en, Magnetic evaluation of fatigue damage in train axles without artificial excitation, Insight 48 (2006), 342-345.

[27] Z.J. Chen, D.C. Jiles et al., Estimation of fatigue exposure from magnetic coercivity, J. Appl. Phys. 75 (1994) 6975.

[28] C.C.H. Lo, F. Tang, D.C. Jiles, S.B. Biner, Evaluation of fatigue damage using a magnetic measurement technique, IEEE Trans. Magn. 35 (1999) 3977.

[29] G.Ya. Bezlyudko, Ye.I. Yolkina, R.N. Solomakha, B.Ye. Popov, Coercivity metering as the basic method of a non-destructive testing of fatigue and as the priority method in the diagnostic set, Int. Conf. ECNDT, Moscow 2010.

[30] M.K. Devine, D.A. Kaminski, L.B. Sipahi, D.C.Jiles, Detection of fatigue in structural steels by magnetic property measurements, J. Mat. Eng. and Perf., 1 (1992) 249-253. 
[31] C.C.H. Lo, F. Tang, S.B. Biner, D.C. Jiles, Effects of fatigue-induced changes in microstructure and stress on domain structure and magnetic properties of $\mathrm{Fe}-\mathrm{C}$ alloys, J. Appl. Phys., 87 (2000) 6520

[32] I. Tomáš, J. Kadlecová, G. Vértesy, B. Skrbek, Investigation of structural modifications in ferromagnetic materials by Magnetic Adaptive Testing, Proceedings of ICBM8 (2010) 153-162.

[33] I. Tomáš, G. Vértesy, Magnetic Adaptive Testing, In: Nondestructive Testing Methods and New Applications, M.Omar (Ed.), ISBN: 978-953-51-0108-6, (2012), InTech: $\quad$ http://www.intechopen.com/articles/show/title/magnetic-adaptivetesting

[34] O. Kováŕík, J. Siegl, J. Nohava, P. Chráska, "Young's Modulus and Fatigue Behavior of Plasma Sprayed Alumina Coatings" In: Thermal Spray 2003: Advancing the Science and Applying the Technology, (Ed.) C.Moreau amd B.Marple, Published by ASM International, Materials Park, Ohio, USA, 2003

[35] O. Kovárík, J. Siegl, and Z. Procházka, Fatigue Behavior of Bodies with Thermally Sprayed Metallic and Ceramic Deposits, J. Thermal Spray Technology 17 (2008) 525-532.

[36] R. Becker, M. Kersten, Die Magnetisierung von Nickeldraht unter starkem Zug, Z.Phys., 64 (1930) 660-681

[37] D. C. Jiles, "Theory of the magnetomechanical effect," J. Phys. D, Appl. Phys., 28 (1995] 1537.

[38] I. Altpeter, G. Dobmann, M. Kroning, M. Rabung, S.Szielasko, Micro-magnetic evaluation of micro residual stresses of the IInd and IIIrd order, NDT\&E International 42 (2009) 283-290

[39] I. Tomáš, J. Kadlecová, G. Vértesy, Measurement of flat samples with rough surfaces by Magnetic Adaptive Testing, IEEE Trans. Magn. 48 (2012) 1441-1444.

[40] I. Tomáš, O. Kováŕík, J. Kadlecová, G. Vértesy, Optimization of fatigue damage indication in ferromagnetic low carbon steel, to be presented at the International Conference on Fatigue Damage of Structural Materials, The Resort and Conference Center at Hyannis, MA, USA, 2014

[41] G. Vértesy, I. Tomáš, I. Mészáros, Investigation of experimental conditions in Magnetic Adaptive Testing, J. Magn. Magn. Mater. 315 (2007) 65-70. 